\title{
Implementando la nueva gestión pública: problemas y desafíos a la ética pública. El caso chileno
}

\author{
Implementing the new public management: problems and challenges to \\ public ethics. The Chilean case
}

\author{
Cristian Pliscoff-Varas / cpliscof@iap.uchile.cl \\ Universidad de Chile, Chile
}

\begin{abstract}
This article analyzes the impact of the implementation of New Public Management doctrines in Chilean public administration. In this work, three administrative doctrines are presented and linked with three corruption scandals that took place in Chile. The research strategy is qualitative, using a case study approach for data gathering. This paper is aimed at exploring the potential connection between the implementation of these new administrative doctrines and the emergence of deeds that are at odds with public ethics. After presenting these three cases, three alternative solutions to those newly adapted doctrines are presented, which are a better prescription regarding public agencies' performance in the region.
\end{abstract}

Key words: New Public Management, public management modernization, corruption, Chile, case studies.

Resumen: El presente documento analiza el impacto de la implementación de algunas doctrinas administrativas propias de la Nueva Gestión Pública (NGP) en la administración pública chilena. En este trabajo se exponen tres doctrinas dominantes de la NGP, relacionándolas con tres hechos de corrupción acaecidos en Chile, por lo cual la estrategia de investigación es cualitativa, utilizando el método del estudio de casos como recurso metodológico. Al hacer esto, se pretende tratar de explorar si pudiese existir una relación entre la aplicación de estas nuevas doctrinas y el surgimiento de actos reñidos con la ética pública. Luego de la descripción de los tres casos, se plantea la relación que éstos tienen con alguno de los modelos teóricos alternativos a la NGP que pudiesen abordar de mejor forma el funcionamiento de la administración pública en la región.

Palabras clave: Nueva Gestión Pública, modernización de la gestión pública, corrupción, Chile, estudio de casos. 


\section{Introducción ${ }^{1}$}

Mucho se ha escrito desde que en 1991 se empezó a acuñar y utilizar el concepto de la Nueva Gestión Pública (NGP) o New Public Management (NPM) en el clásico texto de Hood (1991) y con el trabajo de Aucoin (1990). La llegada de esta nueva forma de entender la gestión pública ha sido materia de revisión a nivel global por diversos actores y a partir de diversas perspectivas (Lane, 2000; Kettl, 2000; Ventriss, 2000; McLaughlin et al., 2002; Lynn, 2006). En esta revisión se ha puesto de manifiesto que existen nuevas ideas que han llegado a determinar cómo se administran o gestionan las instituciones públicas, para aggiornar las administraciones públicas, logrando de esta manera, supuestamente, una mejor gestión. Del mismo modo, se presentan las condiciones en su implementación y potenciales costos.

En el caso de América Latina, la literatura académica nos ha entregado una serie de trabajos que van desde una suerte de declaración de muerte de este paradigma (Guerrero, 2009), hasta otros que plantean que muchos de los cambios generados en nuestras administraciones públicas en el último tiempo, no necesariamente tienen una justificación o relación con la "moda" de la NGP (Cejudo, 2008). El aporte de estas posturas es que han puesto de manifiesto que no se puede mantener una actitud ingenua respecto de un paradigma, esencialmente generado en los países desarrollados con historias y culturas administrativas muy diferentes.

Lo interesante para quien se preocupa por las temáticas propias de la gestión pública es que a pesar de las aprensiones respecto de la real expresión de la NGP en América Latina, muchas de las doctrinas administrativas propias de esta corriente se han implementado en nuestras administraciones públicas (Atrio y Piccone, 2008; Manning y Shepherd, 2009; Dussauge, 2009). Esto ocurre en parte porque los tiempos de la academia no son necesariamente los mismos que los de los diseñadores de política o tomadores de decisiones en el gobierno. Las visiones críticas de las modificaciones no necesariamente son captadas por quienes buscan mejorar el rendimiento de las gestiones públicas, también llamados "practitioners".

Vemos cómo muchos de los conceptos propios de este nuevo paradigma están presentes en el día a día de la gestión del Estado. La legitimidad de estos cambios se sustenta en la necesidad de modificar cómo se hacen las cosas en la gestión pública, adecuando estas prácticas a la nueva realidad social, económica y cultural (Barzelay, 1992). Como plantea Arellano (2002: 58), "la

1 El presente documento da cuenta de parte de los resultados obtenidos en el marco del proyecto de investigación Fondecyt Iniciación núm. 11110207 realizado por el autor. 
NGP es en realidad una propuesta que se explica a la luz de las consecuencias de la evolución y crisis de las formas específicas de accionar de la administración pública y su relación con el sistema político". Se busca un Estado más eficiente y cercano a las necesidades de los usuarios vistos como clientes en búsqueda de mejor atención.

Pero en esta promesa de un mejor Estado, a partir de una mejor administración pública, se imponen ideas que pueden ser muy complejas en contextos administrativos débiles o con culturas administrativas poco consolidadas, proclives a las malas prácticas administrativas e incluso a la corrupción. Ramió (2001) nos plantea un esquema respecto de las potenciales implicancias que conllevaría la adopción de estas nuevas ideas en diferentes contextos administrativos. Esto nos puede llevar a pensar respecto de la potencial relación que pudiese existir entre la adopción de estas nuevas doctrinas administrativas y la ocurrencia de vicios o problemas en la gestión.

El presente documento se orienta a analizar la implementación de la NGP en América Latina, y su relación con la ocurrencia de hechos de corrupción, tomando a la realidad administrativa chilena como el marco de análisis. En este sentido, seguimos la línea planteada por Maesschalck (2004), quien analiza el impacto de la NGP en los marcos éticos de los funcionarios públicos, a partir de la adopción de nuevas doctrinas administrativas, ajenas al paradigma burocrático. Un grupo de casos de corrupción fueron elegidos, debido a que se relacionan con alguna de las doctrinas administrativas de esta filosofía administrativa pública. Una vez efectuado este análisis, se entregarán reflexiones sobre los dilemas teóricos y las aproximaciones que se han propuesto para dar cuenta de ellas.

\section{Nueva Gestión Pública en Chile}

Como parte del análisis realizado para dar cuenta de los objetivos de este documento, resulta necesario reflexionar respecto de la afirmación de si la NGP se ha implementado o no en Chile. Si bien esto puede ser materia de gran debate, producto de lo complejo que resulta reconocer el paradigma burocrático dominante en un país, es necesario reconocer que el proceso de modernización de la gestión pública, iniciado a mediados de la década de 1990, tiene rasgos esencialmente propios del discurso de la NGP, como se puede apreciar en varias investigaciones a este respecto (Ramírez, 2004; Waissbluth e Inostroza, 2006; Araya y Cerpa, 2009; Pliscoff, 2009b; Olavarría, 2010; Olavarria et al., 2011; Figueroa-Huencho et al., 2011; Morales, 2014). 
Se debe hacer especial mención en términos de tres hitos que expresan claramente el "discurso" de la NGP en Chile. El primero guarda relación con el desarrollo de un Plan Estratégico de Modernización de la Gestión Pública, implementado entre 1997 y 2000, donde se define una agenda de cambios en diversos ámbitos de la gestión pública, tales como mejora de los sistemas de planeación, la instalación de mejores mecanismos de control de gestión y la medición del quehacer de las instituciones públicas.

Este esfuerzo inicial se ve complementado y reforzado con dos hitos centrales en el proceso de modernización, la instalación de un sistema de evaluación y control de gestión por parte de la Dirección de Presupuestos (DIPRES) del Ministerio de Hacienda, el cual se instala en 2001, y la creación del Sistema de Alta Dirección Pública en 2003, que empieza a funcionar en 2004, en el marco de la creación de la Dirección Nacional de Servicio Civil.

Tomando las doctrinas administrativas propias de la NGP propuestas por Hood (1991), se puede reconocer claramente que en Chile se han efectuado cambios en las prácticas administrativas públicas en los siguientes aspectos. Primero, ante la idea de "dejar que los gestores gestionen", propuesta por Hood, en el caso chileno se ha implementado por ejemplo, un Sistema de Alta Dirección Pública, que busca reforzar el rol central del directivo público en el desarrollo, instalando un discurso sobre el tema.

En segundo lugar, se han tomado medidas administrativas explícitas para medir el desempeño y controlar la acción de las instituciones públicas, con mecanismos de diverso tipo, como el sistema de evaluación y control de gestión, implementado por la DIPRES. Junto con estas doctrinas, resulta claro el uso intensivo de la tecnología y una referencia a las técnicas privadas de gestión, como por ejemplo, con la implementación de los Programas de Mejoramiento de la Gestión (PMG) en el año 1998, que instalaron la lógica de la remuneración variable por desempeño, tan propia de la gestión privada (Pliscoff, 2005).

\section{Algunas doctrinas dominantes de la NGP}

Con el propósito de profundizar el análisis de las doctrinas dominantes de la NGP en Chile, se tomará como referente el trabajo de Hood (1991) para reconocer tres que son particularmente propias de este paradigma, y que como ya fue expuesto, se han implementado en el marco del proceso de modernización de la gestión pública en Chile. Estas doctrinas, claramente, son muy diferentes de las aproximaciones previas de la administración pública progresiva o burocrática. 
La primera es la contractualización como herramienta de gestión para la provisión de servicios o tareas previamente llevadas a cabo por entidades o funcionarios públicos. La segunda es la idea de "dejar que los gestores gestionen", que en términos prácticos se puede traducir en el incremento en los niveles de discrecionalidad de los gestores públicos. Finalmente, otro de los puntos centrales de la NGP es la presión por resultados, siendo uno de los instrumentos de la gestión predilectos el uso de incentivos para alcanzar esos resultados.

\section{Contractualización}

Durante la década de 1980, la disminución de los ingresos y los elevados costos en los servicios productivos alentaron a los gobiernos a implementar medidas en busca del uso eficiente de los recursos. Dentro de estas innovaciones está la figura de la subcontratación o externalización. En la externalización, los organismos públicos establecen un contrato con una empresa privada para entregar un producto o servicio público, y ésta tiene la función de supervisar dicha entrega.

Esta figura es ampliamente utilizada, por ejemplo, para la recolección de basura, el transporte, servicios administrativos, etcétera. El espíritu de este dispositivo de la NGP es el ahorro en la estructura de costos de los servicios públicos, y además permitiría mejorar los conocimientos técnicos, compartir los riesgos, dar respuestas más rápidas a las necesidades ciudadanas (Hood, 1991).

Sin embargo, la figura de la NGP ha despertado una serie de cuestionamientos, ya que se plantea desde la academia que no todo ámbito de gestión pública puede ser sujeto de externalización (Meier y Hill, 2005). Diferentes actores sugieren que se puede generar competencia por la prestación o entrega de un servicio, creándose un proceso hacia la eficiencia en el uso de recursos (Savas, 2000). No obstante, los detractores de la contractualización afirman que la externalización tiende a separar la formulación de las políticas públicas (fase política) y la producción de éstas (fase administrativa), con lo cual se abren espacios para una reducción del control en la entrega de los servicios públicos que recibe la ciudadanía (Johnston \& Seidenstat, 2007; Arellano, 2002).

Lo contradictorio de este punto es que la contractualización pudiese ser una paradoja, donde por tratar de limitar la capacidad de los burócratas de actuar sin control ni accountability, se pase a una situación donde los privados sean los que no responden a ningún tipo de control ni responsabilidad por lo que hacen o dejan de hacer (Meier y Hill, 2005: 56). 


\section{Discrecionalidad}

Bajo el lema "Dejar que los gestores gestionen", la NGP buscó liberar al gerente público de barreras burocráticas innecesarias, y ampliar el espacio de libertad de gestión de los directivos. Como explica Ingraham (2005: 528), el sistema de personal público ha sido construido dentro de un complejo mundo de reglas y procesos legales y burocráticos, lo que ha decantado en importantes barreras para los directivos públicos. Para superar esto, dentro de la NGP surgen políticas tanto de personal como de gestión pública en general, orientadas a dar más espacio a la discreción en la toma de decisiones.

Esta discreción puede ser para contratar un nuevo funcionario, generar un nuevo servicio, o simplemente, para facilitar la gestión del directivo superior. En esta línea argumental, Moore (1998) centró su análisis en la necesidad de dotar a los directivos públicos de la capacidad para adecuar el uso de los recursos organizacionales y generar "valor público". En su esquema, se plantean tres limitantes a esta creación de valor en las organizaciones públicas: que las decisiones sean legal y administrativamente sustentadas, que tengan apoyo político y que sean valoradas por la ciudadanía. Dotar al directivo público de mayor discrecionalidad se expresa, por ejemplo, en que el directivo podrá determinar la actualización de mandatos y, por ende, definir el norte de una organización.

Las estrategias orientadas a aumentar la discrecionalidad en el personal del sector público, particularmente en los directivos públicos, no han estado exentas de críticas. Dentro del flanco de los cuestionamientos se esboza que incrementar la discrecionalidad puede dejar de lado cosas importantes para la organización, como la memoria histórica, el know how respecto de una materia, y todos aquellos que guarden relación con la capacidad institucional desarrollada con el tiempo (Terry, 1995; Frederickson, 1997).

Por cierto, otro de los problemas que genera el incremento de la discrecionalidad de los funcionarios públicos es la posibilidad de que a menores controles, se aumenten las posibilidades de corrupción o mala administración; porque el aumento de la discrecionalidad se puede dar en un potencial marco de erosión de valores públicos (Moynihan, 2008). Esto produce que quienes poseen esta discrecionalidad no la ejercen de acuerdo con los valores o el ethos que debería regir al servicio público.

Los trabajos de Gregory (1999) y Norman (2003), referidos a los resultados de años de reformas en Nueva Zelanda, ayudan a justificar estos temores respecto de incrementar la discrecionalidad en el sistema público. 
La paradoja se estructura en torno a que por liberar de ataduras y controles al directivo, se desconecta el quehacer de éste con el espacio donde ejerce su gestión. La eficiencia o eficacia en sí misma no sirven si no hay un marco de servicio público observado.

\section{Gestión por resultados}

Siguiendo con lo planteado en el texto base de las doctrinas administrativas de la NGP, Hood (1991) indica que esta nueva visión de la gestión de las organizaciones públicas se centra en un mayor control en los resultados por sobre los procedimientos que justifiquen una determinada tarea. En esta misma línea argumentativa, Barzelay (1992) señala que el quehacer de la administración pública no puede sustentarse en los procedimientos que ésta lleva a cabo, sino en los servicios o productos que se pretenden proveer con un grupo de recursos disponibles.

La centralidad en los procesos propuesta por Weber (1992), se justificaba en el reconocimiento de la llamada racionalidad instrumental, que ponía énfasis en los procedimientos como medios para el logro de determinados fines. Pero tal como ya lo indicó Merton (1999) en su análisis de las disfunciones de la burocracia, debido a las propias características del modelo burocrático se produce un desplazamiento de metas, lo cual lleva a que los medios, vale decir los procedimientos, se transformen en un fin en sí mismo.

La primacía de los resultados en la NGP nos lleva a otros aspectos que acarrean la nueva gestión de las entidades públicas. Como lo señalan Echevarria y Mendoza (1999: 35-36), “(...) bajo el nuevo marco de relaciones, a las agencias, centros o entidades autónomas se les delegan, según sus circunstancias, facultades de gestión de recursos y actividades, lo cual se controla con la estandarización de objetivos y la medición de resultados, normalmente a través de indicadores cuantitativos". La centralidad de los resultados no es sólo un motor orientador de la gestión pública, sino también un medio para poder controlar a quienes tienen niveles mayores de discrecionalidad. El problema se produce en las agencias cuyo quehacer no necesariamente es fácilmente cuantificable.

Presentado de esta manera el debate, a veces se hace difícil la aplicación de esta doctrina en los contextos públicos. La necesidad de reconocer y validar resultados de un determinado servicio público acarrea una presión por la medición, que permita asegurar el logro de esos resultados prometidos. El interés de académicos y practitioners se mueve hacia lo cuantificable, desplazando a otros aspectos difíciles de evidenciar a través de un valor o razón. 
Lo que la literatura ha presentado junto con la presión por cuantificar es la presión por alcanzar el resultado. Se genera un nuevo vicio, ya no de la burocracia, a la Merton, sino un vicio de la NGP, toda vez que se plantea la centralidad del resultado como sentido del quehacer público. En esta línea, Dobel (2005) señala que el centro del ethos público administrativo debe estar focalizado en la ética de la "policy", reconociendo el sentido de lo público en su quehacer profesional antes que centrarse en obtener los incentivos económicos como el centro del ethos. Pliscoff (2005), analizando el caso chileno, señala que existe una visión divergente sobre el valor de los incentivos en la gestión pública, producto de los espacios que dejan para que se presenten casos de corrupción.

Esto se explica por la borrosa estructura en la cual se establecen los incentivos, debido principalmente a la complejidad técnico-administrativa que envuelve el diseño, ocasionando que el accountability sea complejo. Además de lo anterior, se podría generar un problema con la estructura de incentivos, ya que no estaría alineada con el propósito de un mejoramiento en la gestión interna de los servicios públicos.

Como vemos, cada una de estas doctrinas ha generado "áreas grises" o espacios que han llevado a plantear una potencial relación entre ellas en este nuevo paradigma y la ocurrencia de casos de corrupción. Con esto no se está planteando que la NGP per se genere corrupción, sino que las propias doctrinas que se pregonan para lograr un mejor estado pudiesen estar generando las condiciones, para que, en la medida que se junten éstas con individuos carentes de marcos éticos sólidos, pudiesen darse las condiciones para una mayor ocurrencia de hechos de corrupción. La siguiente sección del presente documento pretende indagar en esta posible relación entre doctrinas administrativas de la NGP y algunos casos de corrupción.

\section{Corrupción y gestión pública en América Latina}

El tema de la corrupción ha sido un tema casi omnipresente en la discusión pública en América Latina en las últimas décadas (Weyland, 1998). Las cifras entregadas por Transparencia Internacional, ubican a la región, salvo algunos casos especiales, como una de las que tienen mayor percepción de corrupción en la sociedad. Usualmente, el debate sobre la corrupción gira en torno a la relación entre política y corrupción, cuestión que nos trae a la mente inmediatamente los casos de Fujimori, Collor de Melo, Abdalá Bucaram y otros tantos altos mandatarios involucrados. Como lo señalan Morris y Blake (2009), desde el retorno a la democracia en los ochenta, el tema de la corrupción ha estado 
lamentablemente presente en prácticamente toda la región. Esto, porque de alguna manera se asumió que la ola democratizadora y los supuestos éxitos económicos pos reformas estructurales tendrían que haber generado niveles menores de corrupción, cuestión que de acuerdo con la revisión de la literatura y la experiencia propia de los ciudadanos latinoamericanos dista de ser así (Rehren, 2009).

Pero más allá de los análisis periodísticos o incluso casuísticos sobre el particular, resulta interesante que el tema de la investigación en corrupción, desde un punto de vista académico, ha estado, más bien, ausente del debate. Son pocos los trabajos científicos que se han presentado en esta materia, no sólo por lo complejo de su estudio, sino también porque en estas materias se combinan mucho los discursos normativos con los propiamente empíricos o fácticos.

En esta línea de análisis, Morris y Blake (2009) ordenan la literatura académica en la materia en torno a tres grandes discusiones: las influencias institucionales de la corrupción, el rol de la cultura y la opinión pública, y los esfuerzos y medidas contra la corrupción. Una revisión de los últimos doce años de las dos revistas de mayor prestigio en nuestro ámbito en la región, Reforma y Democracia y Gestión y Politica Pública, nos muestra que si bien este interés académico está presente, no resulta dominante ni mucho menos proporcionalmente mayoritario (Gorrochategui, 2000; Caiden, 2004; Villoria, 2006; Merat y Deubel, 2006; Berthin, 2008; Martínez-Cousinou y Andersson, 2009; Heller, 2009; Pliscoff, 2009a; Peña-Mancillas, 2011; Vertiz, 2000; Mohabbat, 2001; Anechiarico, 2010).

El análisis se hace más difícil cuando tratamos de focalizar nuestro estudio en la relación existente entre administración pública y corrupción; porque de alguna manera se ha asumido, tal como se ve reflejado en la literatura ya presentada, que la gran fuente de corrupción es la política. Pero, ¿qué ocurre cuando la fuente de la corrupción es la propia administración pública? ¿Qué circunstancias se deben dar para que ocurra la corrupción, no necesariamente estimulada desde afuera? Se adopta en este análisis el planteamiento clásico de Thompson (1999), que acepta el carácter ético de los funcionarios públicos, renegando de las denominadas éticas de la neutralidad y de la estructura.

Del mismo modo, Caiden (2004) resalta el carácter individual del actor corrupto, quien finalmente incurrirá en un acto reñido con la ética pública, a propósito de alguna justificación que resulte del cálculo del castigo-beneficio, por una práctica aprendida, por la probabilidad de ser detectado en el acto o por alguna de las teorías explicativas de la corrupción (Fernández, 1999). 
Desde el punto de vista organizacional, el proceso que lleva a que la organización sea la generadora de corrupción se denomina "normalización de la corrupción en organizaciones", la cual, de acuerdo con Ashforth y Anand (2003: 3), responde a tres procesos que ellos definen como pilares: 1) institucionalización, 2) racionalización y 3) socialización. El primer proceso guarda relación con adoptar prácticas reñidas con la moral como parte de la rutina organizacional. El segundo se refiere a usar justificaciones socialmente construidas para incurrir en un acto de corrupción. Y como tercero, la socialización es el traspaso de estas conductas a los recién llegados a la organización. Dichas dimensiones se interrelacionan para generar la "corrupción normalizada", como se puede apreciar en la Figura 1. ${ }^{2}$

En las próximas líneas analizaremos algunos casos de corrupción, que nos ayudarán a determinar la probable relación entre doctrinas administrativas y corrupción en Chile.

\section{Tres casos de la realidad chilena}

Los problemas de la contractualización: El caso Wittman-Ilustre Municipalidad de Iquique

En 2003, la Contraloría Regional de Tarapacá (I Región), al realizar una auditoría selectiva a la Unidad de Aseo y Ornato de la Municipalidad de Iquique, encontró graves irregularidades en el cumplimiento y ejecución del contrato denominado "Servicio de Mantención a la Comunidad", efectuado entre la entidad municipal y la empresa contratista Ernesto Wittman Contreras. La relación entre la Municipalidad y la Empresa Wittman se basa en la figura propuesta ampliamente en la literatura de la NGP denominada contractualización con entidades del sector privado para la externalización de la provisión de servicios públicos.

En este caso particular se aseguraba la provisión de personal, por parte del contratista, para los servicios de aseo, áreas verdes, seremías y servicios generales, contra el pago de sueldos y horas extras por parte de la municipalidad. Este tipo de contratos entre el sector público local y organismos del sector privado es muy frecuente, debido a la escasez de recursos económicos y humanos de los municipios para hacer frente a los distintos problemas públicos que deben resolver.

En particular, en este caso se presentan irregularidades durante los años 2000 y 2001, como la aprobación de pagos, por parte de la municipalidad a 2 La figura se encuentran al final del presente documento, en el Anexo (Nota de los editores). 
la empresa, con base en trabajadores que ésta presentaba como si estuvieran prestando los servicios, pero que en realidad o no estaban vinculados con la empresa y en algunos casos se encontraban difuntos. Junto con lo anterior, en el caso que las personas se encontraban realmente en la nómina de empleados de la empresa, ésta canceló sólo parcialmente los sueldos a sus trabajadores. Éstos y otros hechos, especificados en informes de la Contraloría General de República, fueron calificados por la justicia como fraude al fisco y estafa.

No obstante las responsabilidades de la empresa privada, la Contraloría General de la República objetó el accionar de la Municipalidad de Iquique, en especial de los funcionarios de la Unidad de Aseo y Ornato, por no resguardar el patrimonio municipal, ya que no se establecieron los mecanismos de seguimiento y control para evitar que estas irregularidades se cometieran. Claramente, la Municipalidad adoptó la política de contractualización, pero no estableció adecuados mecanismos administrativos para velar por los resultados de la externalización de los servicios, lo cual abre amplios espacios para la germinación de casos de corrupción.

Bajo la figura de la contractualización están los supuestos de la NGP de que los privados resuelven de una forma más eficiente las necesidades de la ciudadanía. En este sentido, a un municipio le resultaría más económico externalizar ciertos servicios públicos que tener en su planta estable personal que brinde solución a estas problemáticas. De esta forma se estaría creando valor público. Sin embargo, la celebración de contratos entre el sector público abre amplios espacios de problemas si no existe una adaptación de la estructura de los servicios públicos hacia una función de fiscalización y robusteciendo los mecanismos de accountability. Se debe tener presente que, según lo que menciona la teoría, los actores del sector privado buscarían maximizar sus beneficios personales antes que maximizar el interés común. Debido a estos propósitos divergentes, es necesario establecer mecanismos de control ad hoc que puedan hacer frente a estas nuevas políticas propuestas por la NGP.

La centralidad de los resultados: El caso de Correos de Chile

El 29 de julio de 2005, en diversos medios, Correos de Chile es denunciado por pérdidas, cobros abusivos y hasta hurto de correspondencia o encomiendas. En una primera instancia las respuestas por parte de los trabajadores frente a los hechos se situaron bajo dos líneas: la gran cantidad de envíos (más 500 mil) y la potente suma de funcionarios externos a la organización. Aproximadamente un año más tarde las pérdidas de correspondencia vuelven a aflorar en diferentes medios de comunicación, y se descubre a tres funcionarios en distintas situaciones destruyendo más de diez mil cartas. 
Una de las principales razones para explicar la quema de cartas subyace en la política de incentivos monetarios al interior de la empresa, ya que se fijó una estructura de pago de diez pesos chilenos por carta, generando un interés pernicioso para obtener más utilidades. Meses más tarde el gerente general de Correos de Chile, Patricio Tapia, explicó que se hizo una auditoría operativa para determinar las responsabilidades que van más allá de la deplorable actuación de los carteros.

El proceso culminó con la desvinculación de tres altos cargos. Se efectuó una auditoría para aclarar los hechos detectando importantes anomalías, indico Patricio Tapia ${ }^{3}$ Gerente General de Correos. Todo indica que no se tomaron a tiempo los resguardos pertinentes, ni se aplicaron los controles para cumplir con la misión institucional en el tiempo y en el modo adecuado. Las declaraciones del gerente general dan muestra del deterioro de la función pública, producto del descuido de los procesos y la centralización en los resultados individuales como resultado de la política de incentivos económicos de la empresa estatal.

Las medidas emprendidas por la gerencia de la empresa fueron apoyadas por el Sindicato de trabajadores de Correos, quienes en sus declaraciones dejaron entrever la responsabilidad de lo acontecido en funcionarios externos a la institución. "Entendemos que una empresa de alrededor de 5.000 trabajadores en algún momento pueda haber algún problema, pero nosotros no nos podemos responsabilizar, la gente de carrera de esta empresa, la gente que somos hijos de funcionarios de Correos de Chile, no nos vamos a responsabilizar de lo que pueda hacer un externo", aseveró el presidente del sindicato.

En lo pronunciado por Martínez no sólo se defiende a la Institución, sino también la dignidad del cargo de funcionario público. Frente a este escenario, el sindicato de trabajadores de Correos de Chile (que agrupaba al 60\% de los carteros del país) planteó la necesidad de crear una superintendencia que regule al sector.

El caso de Correos da cuenta de cómo la convergencia de las doctrinas bases de la NGP, en este caso, una política orientada a obtener resultados específicos y del mismo modo a dar cuenta de una política de incentivos sin considerar la función pública como aspecto central del desempeño profesional, puede generar un escenario propicio para actos de corrupción.

No cabe duda de que el logro de metas y resultados es fundamental para justificar a las organizaciones públicas y muy especialmente para dar cuenta

3 Situación constatada en el periódico La Estrella de Valparaíso, el 4 de octubre de 2006,

"Despidieron a tres ejecutivos de Correos", pág. 9 
de las necesidades que quiere cubrir la sociedad. Lo que nos entrega este caso es que los resultados no pueden estar por delante de la razón básica de ser de una entidad: la entrega de un servicio público. En este caso, esta noción tan básica fue violentada.

\section{Los problemas de la discrecionalidad: El caso de Chiledeportes}

El Instituto Nacional de Deportes se crea con la promulgación de la Ley núm. 19712 del año 2001. En dicha ley se establece su ordenamiento como un servicio público funcionalmente descentralizado (formado por una Dirección Nacional con sede en Santiago, y por Direcciones Regionales de Deportes), dotado de personalidad jurídica y patrimonio propio, vinculado con el Presidente de la República por medio del Ministerio Secretaría General de Gobierno.

En cuanto a su misión, el artículo 11 de la misma ley señala: "Corresponderá al Instituto proponer la política nacional de deportes. Asimismo, tendrá a su cargo la promoción de la cultura deportiva en la población, la asignación de recursos para el desarrollo del deporte y la supervigilancia de las organizaciones deportivas en los términos que establece la presente ley".

El caso "Chiledeportes" tiene sus orígenes en una investigación de la Contraloría General de la República (a petición del senador José García Ruminot, realizada el 25 de enero de 2006), que suponía un presunto desvío de recursos con motivo de las últimas elecciones presidenciales y parlamentarias del año 2005. En dicha investigación se realizó una auditoría a los proyectos financiados con cargo al Fondo Nacional para el Fomento del Deporte (FONDEPORTE) del citado Servicio.

Esta auditoría detectó irregularidades en 70 de 77 proyectos de la institución, cometidas a fines de 2005 , cuyo monto ascendía a los $\$ 417$ millones de pesos. Entre las observaciones de carácter patrimonial producto de la investigación destacaron gastos: sin documentación de respaldo, indebidos o que no corresponden a los objetivos de los proyectos, sin especificar, sin acreditar beneficiarios, respaldados con facturas que presentan situaciones irregulares con el Servicio de Impuestos Internos, entre otras.

Una vez conocido el hecho, comienza una serie de querellas. El 23 de octubre del año 2006 un grupo de seis parlamentarios de Renovación Nacional (RN) (Alberto Espina, José García Ruminot y Nicolás Monckeberg) y de la Unión Demócrata Independiente (UDI) (Andrés Chadwick, Marcelo Forni y Julio Dittborn) interpusieron una querella bajo las causales de "Fraude al Fisco", "Malversación de fondos públicos" y "Uso malicioso de instrumento privado". 
A esta querella se suma la interpuesta por el Consejo de Defensa del Estado en noviembre de 2006, que agregaban los ilícitos de "Falsificación de instrumento público" y "Apropiación indebida de dinero". Los principales acusados del caso fueron el ex Director Regional Metropolitano de Chiledeportes, Juan Michel Salazar, quien fue detenido bajo la acusación de "Fraude al Fisco", y su jefe de Gabinete, David Navarro, también acusado de fraude; a ellos se suma el funcionario del Servicio de Impuestos Internos, Luis Jofré, procesado por "Fraude al Fisco" y Mario Salazar (primo de Juan Michel), declarado culpable por el delito de "Estafa reiterada al Fisco". Entre las principales consecuencias del caso se cuentan modificaciones a la Ley de Deporte núm. 19712 y a la Ley Orgánica del Instituto Nacional del Deporte.

La NGP aboga por aumentar los márgenes de discrecionalidad de los gerentes públicos, bajo el supuesto de que podrán realizar su labor con mayor eficiencia. No obstante, cuando no existe un ethos público internalizado en los funcionarios públicos, podrían ocurrir casos de corrupción. En el caso Chiledeportes, los funcionarios públicos debían seleccionar proyectos para proveer infraestructura deportiva; sin embargo, se asignó una gran cantidad de proyectos sin existir la documentación de respaldo requerida desde un punto de vista técnico.

Junto con esto, se detectó la asignación de recursos a proyectos que presupuestaban gastos que no respondían a los objetivos establecidos por parte del proyecto o la adjudicación de recursos a proyectos donde no se acreditaba quiénes serían los beneficiarios. De lo anterior se puede desprender que el funcionario público al tener amplios espacios de discrecionalidad, en este caso para la entrega de recursos, puede incurrir en casos de corrupción, al no existir al interior de la organización mecanismos de control propicios para detectar estos comportamientos. Al existir espacios no normados en materias en los cuales el funcionario debe decidir, si no existe una ética pública claramente internalizada en él, es probable que tienda a evidenciar conductas corruptas.

\section{Reflexiones de los casos}

Como se observa en cada uno de los tres casos presentados, se pueden apreciar las paradojas expuestas en la sección previa de este trabajo. La doctrina administrativa dominante por tratar de dar cuenta de un fin específico, propio del nuevo marco normativo, puede llevarnos a una situación en que las buenas intenciones generan más problemas. 
En el caso de la contractualización, se puede apreciar como un acto que busca hacer más eficiente el uso de los recursos públicos, genera costos mayores y puede producir problemas de gestión y con la comunidad de real relevancia. Especial interés cobra en el análisis del caso de Iquique uno de los dos mayores problemas en la contractualización, la dificultad de tener contratos perfectos. Las materias de gestión pública son tan complejas que es muy difícil incorporar todas sus potenciales situaciones o excepciones en un documento.

En el caso de las concesiones de construcción y administración de caminos en Chile, por ejemplo, hemos podido ver que los contratos han tenido que ser modificados debido a los imponderables y a las características propias del servicio que se brinda. El caso de Iquique nos muestra que por más simples que puedan ser las funciones que se pretenden externalizar, siempre existe un espacio para la indefinición, lo cual finalmente puede ser utilizado por una u otra parte del contrato.

El segundo gran problema de la contractualización se refiere al grado de accountability de los actores privados. La literatura periodística nos ha mostrado, como en el caso de la pasada guerra en Iraq, la más privatizada de todas las guerras, que los actores privados que estaban cumpliendo funciones públicas prácticamente no eran sujetos de responsabilidad por sus actos (Scahill, 2008). Quien está cumpliendo una función pública, pero tiene un contrato de trabajador privado, ¿a quién debe responder?

En definitiva, como señalan Meier y Hill $(2005,56)$, por tratar de desligarnos de burocracias ineficientes, con agendas propias, estamos creando actores privados que en función de una supuesta eficiencia pueden llegar a ser aún más difíciles de ser responsables por sus actos. El caso de Nueva Zelanda, ya explicitado previamente, resulta interesante en este sentido, ya que es uno de los países con mayor trayectoria de reformas del tipo NGP.

Para el caso sobre la gestión por resultados y la necesidad de medirlos, los cuales eventualmente pueden generarse a partir de la aplicación de incentivos, podemos plantear que la paradoja nos muestra una suerte de línea de continuidad entre lo que planteó Merton (1999), respecto de la burocracia con lo que ocurre con la NGP, a partir del caso expuesto de la empresa de Correos de Chile. Esto porque, tal como señalaba el autor, se origina un desplazamiento de metas en la burocracia, producto del interés de parte de los burócratas de cumplir los procedimientos por sí mismos, pasando a una situación de cumplir los resultados por el indicador, y en el extremo, cumplir los resultados sin miramientos de los procedimientos. 
El hecho de quemar las cartas en una empresa que funda su ethos en el comunicar a todos los chilenos, independientemente del lugar donde se encuentran, no es sólo un tema de desprolijidad o desinterés, es un problema que va a la médula misma de la institución, a su esencia como servicio público.

Finalmente, el análisis del caso Chiledeportes nos lleva a analizar las implicancias de incrementar la discrecionalidad en un contexto de asignación de fondos para proyectos. Se busca de esta manera empoderar al directivo para, en función de cierto nivel de discrecionalidad, asignar recursos con facilidad. Weber (1992), en el modelo burocrático, buscaba limitar a través de la observancia de determinados procedimientos, la discrecionalidad de los directivos públicos, porque se asumía que ese margen permitía acciones erróneas o perjudiciales para la mayoría.

En el marco de la actual doctrina administrativa de la NGP, respecto de "dejar que los gestores gestionen", se repiensa y en cierta manera se vuelve a una etapa donde el directivo define qué se hace y qué no. El problema de incrementar esta discrecionalidad, más allá de los controles que pudiesen haber, se refiere a que los contextos sociales tan disímiles de América Latina impiden que todos tengan la misma posibilidad de ser tomados en cuenta al momento de definir qué se hace o qué no. La discrecionalidad positiva deja de serlo si no hay una conexión con el entorno con altos niveles de transparencia y control ciudadano.

\section{Conclusiones}

Una vez finalizado el análisis propuesto, podemos apreciar que tres doctrinas esenciales de la NGP generan, desde el punto de vista conceptual o teórico, un grupo de paradojas o dilemas, que por tratar de buscar un objetivo terminan generando nuevas realidades, tanto o más complejas que las que se buscaban evitar.

Pero, ¿de qué manera se están abordando estos dilemas en marcos teóricos nuevos o qué están planteando, una nueva visión respecto de los vacíos o áreas grises que se generan a partir de las doctrinas de la NGP? En este marco resulta interesante tomar en consideración tres modelos teóricos que se han planteado principalmente en la academia, y que están siendo concebidos como espacios de reflexión para pretender un nuevo modelo de gestión pública.

Según el modelo propuesto por Kooiman (2004), la gobernanza es entendida como un fenómeno social que cuenta con la participación del gobierno y del sector privado. En este sentido, se entiende el gobierno inte- 
ractivo como "los acuerdos con los que tanto los actores públicos como los privados persiguen solventar problemas sociales o crear oportunidades sociales" (Kooiman, 2004: 2). Así, las fronteras divisorias entre el sector público y el privado se están difuminando cada vez más.

Por lo tanto, existiría un cambio en la relación entre el Estado y el sector privado, transitando desde un modelo unidireccional de gobernantes a gobernados, a uno bidireccional donde se tiene en consideración aspectos, problemas y oportunidades del sistema de gobierno como del sistema a gobernar. En la praxis, la participación del sector privado en conjunto con el sector público ha experimentado un alto crecimiento en las últimas décadas.

En la actualidad presenciamos un notorio cambio de paradigma, desde una visión que encasillaba la resolución de los problemas públicos por parte del Estado, la racionalidad maximizadora de utilidades por parte del sector privado con fines de lucro, y las obras de caridad o filantropía por organizaciones del sector privado sin fines de lucro. Sin embargo, cada vez más aparecen nuevas formas que desafían la rigidez de estas tipologías en la figura de arreglos organizacionales, en los que el sector privado resuelve problemas públicos, y el sector público influye notoriamente en la función de las firmas privadas.

Aunado a esto, surgen las organizaciones privadas sin fines de lucro u ONG que realizan acciones en diferentes etapas del ciclo de política pública específica, comenzando a configurar la esfera de la sociedad civil. A partir de este modelo, los vicios, por ejemplo, de la contractualización quedan de lado toda vez que el actor privado es visto como un actor activo en la solución del problema público. En este sentido, la gobernanza permitiría el desarrollo de dispositivos institucionales como una evolución en pro de una mayor participación en los asuntos públicos, posibilitando un accountability oportuno, reduciendo los espacios de opacidad que pueden ser provocados por las directrices de la NGP.

Otra teoría que se ha planteado en el último tiempo y que ayuda a dar cuenta de uno de los aspectos más débiles de la NGP es el llamado New Public Service (NPS) o Nuevo Servicio Público. Esta aproximación teórica desarrollada por Denhardt y Denhardt (2000), se plantea como una visión diferente a dos extremos, la administración pública tradicional o progresiva y la NGP. Para los autores, el NPS busca poner de manifiesto que los funcionarios públicos no se definen como servidores de un superior jerárquico, ni deben preocuparse de brindarle un determinado servicio a los "clientes" de la gestión pública, sino que deben actuar como articuladores de los diferentes intereses dentro de la sociedad. No dejan de lado el modelo burocrático ni desechan la NGP, focalizan en el marco ético del quehacer público, para jus- 
tificar las doctrinas administrativas a ser tomadas en cuenta por los actores públicos (Maesschalck, 2004).

Como vimos en el caso de la empresa de Correos de Chile, seguir ubicando el análisis de los cambios en la gestión pública con una centralidad excesiva en los resultados, puede llevar a una desconexión de este desempeño con los valores que debería buscar y observar la gestión estatal. El reforzamiento de los marcos éticos para reforzar una nueva relación entre ciudadanos y gestores públicos sitúa el debate de los cambios y de la modernización en un nivel diferente, más acorde con lo que se requiere en la actualidad.

La Administración Pública Posmoderna surge como la antítesis al positivismo rechazando los principales supuestos epistemológicos sobre el comportamiento en las ciencias sociales. La corriente de la Administración Pública Posmoderna se basa en las diferencias entre la modernidad, donde el conocimiento se encontraba basado en la razón, y la propia posmodernidad, la razón lógica, pierde la supremacía. Los posmodernos describen la vida como una hiperrealidad, en la cual ocurre un proceso de destrucción de los significados. Según Baudrillard (1984), los ideales de verdad, racionalidad, certeza y coherencia han perdido su centralidad. Farmer (1995) lleva los supuestos de la posmodernidad a la administración pública, señalando que los preceptos propios de la modernidad permea el andamiaje burocrático, y por lo tanto, al cambiar esos preceptos, debe cambiar necesariamente la propia administración pública.

Los posmodernistas están más cercanos a una crítica abierta al Estado piramidal, rechazando al Estado-nación como constructo teórico y, por ende, negando que los administradores públicos son agentes del Estado y del interés público, resaltando una necesaria relación de éstos con la ciudadanía. Según Frederickson y Smith (2003: 152-153), la administración pública posmoderna señala que los administradores públicos así como las agencias nunca podrán ser neutrales u objetivas. Por lo tanto, la administración pública posmoderna hace énfasis en los equipos de trabajo y las redes, con el fin de reducir la jerarquía de las estructuras.

Esto tiene relación con lo que plantean Fox y Miller (1995), cuando señalan que el foco ha cambiado desde un centro en la burocracia hacia uno centrado en la ciudadanía. La opacidad de la burocracia moderna se modifica a una administración posmoderna gobernada por los diálogos y procesos de transparencia, que permitan fundir al funcionario con el ciudadano en una misma amalgama. La discrecionalidad del directivo en este marco teórico es pensada no desde lo que el directivo considera como pertinente, sino de lo que surja de la relación entre directivo y ciudadanos. 
En este contexto, a modo de recomendaciones de política para dar cuenta de los potenciales problemas que acarrea la implementación de alguna de las doctrinas dominantes de la NGP, se pueden plantear algunas acciones en los siguientes tres ámbitos. Primero, al momento de implementar procesos de contractualización, es fundamental que las oficinas públicas refuercen sus equipos que actúan como contraparte de las entidades privadas que brindan los servicios a la ciudadanía.

Esto implica reforzar las capacidades de fiscalización, ya sea con mejores profesionales o con mejores procedimientos para ese fin, para verificar que el actuar de los privados se ajuste a las necesidades públicas que se pretenden resolver. En segundo lugar, y en una línea muy similar de acción, es clave que los gobiernos refuercen los equipos a cargo de los sistemas de control de gestión, para generar información útil, relevante y pertinente hacia el logro de los fines últimos que se buscan conseguir. Sin un control de gestión adecuado, las instituciones públicas estarán a la deriva en términos del real impacto de su actuación.

Finalmente, es fundamental que los gobiernos articulen una política pública de reforzamiento ético de los funcionarios públicos. Esto implica un proceso de inducción, capacitación y reforzamiento de todos los funcionarios públicos en las materias propias de la ética pública. Junto con ello, se deben actualizar regularmente las normas y procedimientos de forma tal que sirvan para evitar conductas reñidas con la ética pública, y para que refuercen las marcos éticos de quienes ejercen la función pública.

Resulta esencial para darle continuidad a este trabajo, abordar con mayor precisión las dinámicas propias que ha planteado la NGP en términos de otras doctrinas administrativas y, por ende, de otros ámbitos de reformas administrativas, que también pudiesen generar un ambiente propicio para hechos de corrupción. Sería fundamental en ese sentido, profundizar en otros casos de corrupción que pudiesen develar si los cambios en la forma en que está actuando la gestión pública son producto de los nuevos marcos teóricos, o son más bien la expresión de individuos que por su formación o por la ocasión que se genera, llegarán de todas formas a corromperse, independientemente de los marcos organizacionales públicos donde se desarrollan.

Del mismo modo, sería interesante como futuro ámbito de investigación, comparar los casos estudiados en este documento con casos similares en otros contextos administrativos públicos. Con esto se podría ayudar a develar si efectivamente las nuevas doctrinas administrativas ayudan a instalar prácticas perjudiciales para el bien común, o si hay otros factores que explican el hecho de que existan funcionarios que se aparten de un actuar probo. 
Convergencia Revista de Ciencias Sociales, núm. 73, 2017, Universidad Autónoma del Estado de México

\section{Referencias}

Anechiarico, Frank (2010), "La corrupción y el control de la corrupción como impedimentos para la competitividad económica”, en Gestión y Politica Pública, núm. 2, México: CIDE.

Araya, Eduardo y Cerpa, Andrés (2009), "La nueva gestión pública y las reformas en la Administración Pública Chilena”, en Tékhne-Revista de Estudos Politécnicos, núm. 11, Portugal: Instituto Politécnico do Cávado e do Ave.

Arellano, David (2002), "Nueva Gestión Pública: ¿el meteorito que mató al dinosaurio? Lecciones para la reforma administrativa en países como México", en Reforma y Democracia, núm. 23, Venezuela: CLAD.

Ashforth, Blakey Anand, Vikas (2003), "The Normalization ofCorruption in Organizations”, en Kramer, R. M. y Staw, B. M. [eds.], Research in Organizational Behavior, núm. 25, Estados Unidos: JAI Press.

Atrio, Jorge y Piccone, María Sol (2008), “De la administración pública a la gerencia pública: El porqué de la necesidad de gestionar la transición”, en Reforma y Democracia, núm. 42, Venezuela: CLAD.

Aucoin, Peter (1990), "Administrative Reform in Public Management: Paradigms, Principles, Paradoxes and Pendulums", en Governance, vol. 3, núm. 2, Reino Unido: Blackwell Publishing.

Barzelay, Michael (1992), Breaking Through Bureaucracy: A New Vision for Managing in Government, Estados Unidos: University of California Press.

Baudrillard, Jean (1984), "Interview: Games with Vestiges", en On the Beach 5, invierno, Reino Unido.

Berthin, Gerardo (2008), "Fortalecimiento de la capacidad de formular e implementar políticas de transparencia y anticorrupción en América Latina”, en Reforma y Democracia, núm. 41, Venezuela: CLAD.

Caiden, Gerald (2004), "El peso que cargamos sobre nuestras espaldas: corrupción en América Latina”, en Reforma y Democracia, núm. 28, Venezuela: CLAD.

Cejudo, Guillermo (2008), "Explaining Change in the Mexican Public Sector", en International Review of Administrative Sciences, num. 74, vol. 1, Inglaterra: Sage.

Denhardt, Robert y Denhardt, Janet (2000), “The New Public Service: Serving Rather than Steering”, en Public Administration Review, núm. 60, vol. 6, Estados Unidos: WileyBlackwell.

Dobel, J. Patrick (2005), "Public Management as Ethics", en Ferlie, Ewan et al. [comp.], The Oxford Handbook of Public Management, Estados Unidos: Oxford University Press.

Dussauge, Mauricio (2009), "¿Todos los caminos llevan a la nueva gestión pública? Tres argumentos sobre las reformas administrativas en los países en desarrollo/transición”, en Revista Estado, Gobierno y Gestión Pública, núm. 13, Chile: INAP.

Farmer, David J. (1995), The Language of Public Administration: Bureaucracy, Modernity, and Postmodernity, Estados Unidos: University Alabama Press.

Echevarria, Koldo y Mendoza, Xavier (1999), "La Especificidad de la Gestión Pública: el Concepto de Management Público”, en Losada i Madorrán [ed.], ¿De Burócratas a Gerentes?, Estados Unidos: Banco Interamericano de Desarrollo.

Fernández, Luis (1999), Psicología de la corrupción y los Corruptos. Teorías explicativas de la corrupción, España: Universidad de Santiago de Compostela. 
Cristian Pliscoff-Varas. Implementando la nueva gestión pública: problemas y desafios a la ética pública.

Figueroa-Huencho et al. (2011), "Política de Modernización de la Gestión Pública en Chile 1990-2006: evidencias a partir de un modelo de análisis”, en Convergencia, Revista de Ciencias Sociales, vol. 18, núm. 57, México: Universidad Autónoma del Estado de México.

Fox, Charles y Miller, Hugh (1995), Postmodern Public Administration: Toward Discourse, Estados Unidos: Sage.

Frederickson, H. George y Smith, Kevin (2003), The Public Administration Theory Premier, Estados Unidos: Westview Press.

Frederickson, H. George (1997), The Spirit of Public Administration, Estados Unidos: Jossey-Bass.

Gorrochategui, Nora (2000), “Medidas preventivas de la corrupción en el funcionamiento del aparato público”, en Reforma y Democracia, núm. 17, Venezuela: CLAD.

Gregory, Robert (1999), "Social capital theory and administrative reform", en Public Administration Review, núm. 59, Estados Unidos: Wiley-Blackwell.

Guerrero, Omar (2009), "El fin de la nueva gerencia pública”, en Revista Estado, Gobierno y Gerencia Pública, núm. 13, Chile: INAP.

Heller, Nora (2009), "Definiendo y midiendo la corrupción. ¿De dónde venimos, dónde estamos ahora y qué importa para el futuro?", en Reforma y Democracia, núm. 45, Chile: INAP.

Hood, Christopher (1991), “A Public Management For All Seasons?”, en Public Administration, num. 69, vol. 1, Reino Unido: Blackwell Publishing.

Ingraham, Patricia (2005), "Striving for Balance: Reform in Human Resource Managemement", en Ferlie, Ewan et al. [comp.], The Oxford Handbook of Public Management, Estados Unidos: Oxford University Press.

Johnston, Van y Seidenstat, Paul (2007), “Contracting out government services: privatization at the millennium”, en International Journal of Public Administration, num. 30, vol. 3, Reino Unido: Taylor and Francis Inc.

Kettl, Donald (2000), The Global Public Management Revolution: A Report on the Transformation of Governance, Estados Unidos: Brookings Institution.

Kooiman, Jan (2004), “Gobernar en Gobernanza”, en Revista Instituciones y Desarrollo, núm. 16, España: Institut Internacional de Governabilitat de Catalunya.

Lane, Jan Erick (2000), New Public Management, Estados Unidos: Taylor \& Francis.

Lynn, Lawrence (2006), Public Management: Old and New, Estados Unidos: Routledge.

Maesschalck, Jeroen (2004), "The impact of new public management reforms on public servants' ethics: Towards a theory”, en Public Administration, núm. 82, vol. 2, Reino Unido: Blackwell Publishing.

Manning, Nick y Shepherd, Geoffrey (2009), "Reforma de la Gestión Pública ¿Qué debe aprender América Latina de la OCDE”, en Reforma y Democracia, núm. 44, Venezuela: CLAD.

Martínez-Cousinou, Gloria y Andersson, Staffan (2009), "El control institucional de la corrupción: un marco analítico para su estudio”, en Reforma y Democracia, núm. 43, Venezuela: CLAD.

McLaughlin, Kathleen et al. (2002), New Public Management: Current Trends and Future Prospects, Reino Unido: Routledge.

Meier, Kenneth e Hill, Gregory (2005), "Bureaucracy in the Twenty first century", en Ferlie, Ewan et al. [comp.], The Oxford Handbook of Public Management, Estados Unidos: Oxford University Press. 
Merat, Jacques y Deubel, André-Noël Roth (2006), "La efectividad de las instituciones en la lucha contra la corrupción: la estrategia de los Pactos de Transparencia en Colombia", en Revista Reforma y Democracia, núm. 36, Venezuela: CLAD.

Merton, Robert (1999), "La estructura burocrática y la personalidad”, en Jay, M. y Hyde, Albert, Clásicos de la Administración Pública, México: Fondo de Cultura Económica.

Mohabbat, Mohammad (2001), "Problemas de la Democracia: Reforma Administrativa y Corrupción”, en Gestión y Politica Pública, núm. 9, México: CIDE.

Moore, Mark (1998), Gestión Estratégica y Creación de Valor en el Sector Público, España: Paidós.

Morales, Marjorie (2014), "Nueva Gestión Pública en Chile”, en Revista de Ciencia Politica (Santiago), vol. 2, núm. 34, Chile: Pontificia Universidad Católica de Chile.

Morris, Stephen D. y Blake, Charles H. (2009), "Political and Analytical Challenges of Corruption in Latin America”, en Blake, Charles H. y Morris, Stephen [eds.], Corruption \& Democracy in Latin America, Estados Unidos: University of Pittsburgh Press.

Moynihan, D. P. (2008), "The normative model in decline? Public service motivation in the age of governance”, en Perry, James y Hondeghem, Annie [eds.], Public service motivation: State of the science and art, Estados Unidos: Oxford University Press.

Norman, Richard, (2003), Obedient servants?, Canadá: Victoria University Press.

Olavarría-Gambi, Mauricio (2010), "Efectividad en la gestión pública chilena", en Convergencia, Revista de Ciencias Sociales, vol. 17, núm. 52, México: Universidad Autónoma del Estado de México.

Olavarría-Gambi, Mauricio et al. (2011), “¿ Cómo se formulan las políticas públicas en Chile?: Evidencia desde un estudio de caso”, en Politica y gobierno, vol. 1, núm. 18, México: CIDE.

Peña-Mancillas, Víctor (2011), "Combatir la corrupción en el Perú: a diez años de Fujimori”, en Reforma y Democracia, núm. 51, Venezuela: CLAD.

Pliscoff, Cristian (2005), "Sistema de Incentivos Monetarios y Reforma del Estado: Elementos para una discusión necesaria”, en Documentos de Apoyo Docente. Escuela de Gobierno y Gestión Pública, Chile: Universidad de Chile.

Pliscoff, Cristian (2009a), "Explorando el tipo de razonamiento moral de los funcionarios públicos chilenos", en Reforma y Democracia, núm. 45, Venezuela: CLAD.

Pliscoff, C. (2009b), New public management in Chile (1990-2008): exploring its impact on public employees (Doctoral dissertation), Estados Unidos: University of Southern California.

Ramió, Carlos (2001), “Los Problemas de la Implantación de la Nueva Gestión Pública en las Administraciones Latinas: Modelo de Estado y Cultura Institucional”, en Reforma y Democracia, núm. 21, Venezuela: CLAD.

Ramírez, Álvaro (2004), El proceso de reforma del Estado y modernización de la gestión pública en Chile: lecciones, experiencias y aprendizajes (1990-2003), España: Instituto Nacional de Administración Pública (INAP).

Rehren, Alfredo (2009), “The Crisis of Democratic State”, en Blake, Ch. y Morris, S. [eds.], Corruption \& Democracy in Latin America, Estados Unidos: University of Pittsburgh Press.

Savas, E. S. (2000), Privatization and public-private partnerships, Estados Unidos: Chatham House Pub. 
Cristian Pliscoff-Varas. Implementando la nueva gestión pública: problemas y desafios a la ética pública.

Scahill, Jeremy (2008), Blackwater: The Rise of the World's most Powerful Mercenary Army, Estados Unidos: Nation Books.

Terry, L. Douglas (1995), Leadership of Public Bureaucracies: The Administrator as Conservator, Estados Unidos: Sage Thousand Oaks.

Thompson, Dennis (1999), "La posibilidad de una Ética Administrativa”, en Shafritz, J. y Hyde, A., Clásicos de la Administración Pública, México: Fondo de Cultura Económica.

Transparencia Internacional (2015). Disponible en: http://www.transparency.org/ cpi2015 [1 de febrero de 2016].

Ventriss, Curtis (2000), "New Public Management: An Examination of Its Influence on Contemporary Public Affairs and Its Impact on Shaping the Intellectual Agenda of the Field", en Journal of Administrative Theory and Praxis, núm. 22, vol. 3, Estados Unidos: M. E. Sharpe.

Vertiz, Miguel Ángel (2000), “El Combate a la Corrupción en los trámites administrativos: una aproximación teórica”, en Gestión y Politica Pública, núm. 9, México: CIDE.

Villoria, Manuel (2006), La corrupción politica, España: Síntesis.

Waissbluth, Mario e Inostroza, José (2006), "La reforma del Estado en Chile 1990-2005. De la confrontación al consenso", en ESADE y Departamento de Ingeniería Industrial, Chile: Universidad de Chile.

Weber, Max (1992), Economia y Sociedad: Esbozo de sociología comprensiva, Argentina: Fondo de Cultura Económica.

Weyland, K. G. (1998), "The politics of corruption in Latin America”, en Journal of Democracy, vol. 9, núm. 2, Estados Unidos: The Johns Hopkins University Press.

\section{Anexo}

\section{Figura 1}

\section{Los tres pilares de la normalización}

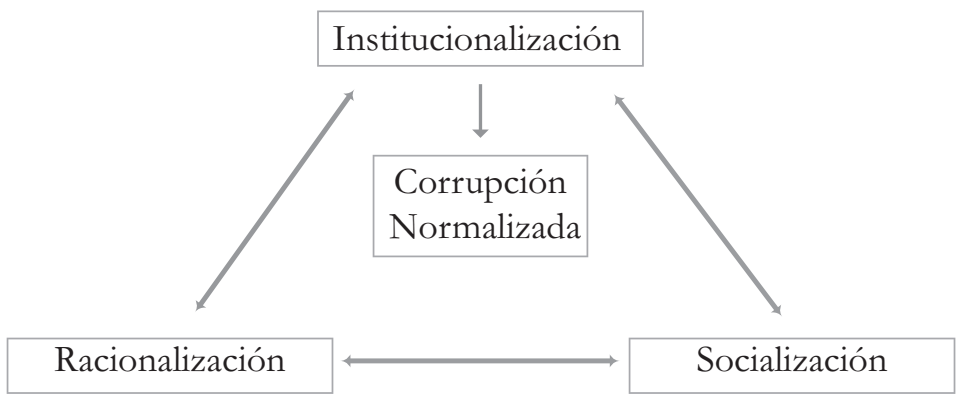

Fuente: Ashford y Anand (2003: 3). 
Cristián Pliscoff-Varas. Profesor asociado, Universidad de Chile. Administrador público, Universidad de Chile. Máster en Gestión y Políticas Públicas, London School of Ecnomics. Ph.D. Public Administration, University of Southern California. Director Magíster en Gobierno y Gerencia Pública, Instituto de Asuntos Públicos, Universidad de Chile. Presidente, Red Interamericana de Educación en Administración Pública (INPAE) (2016-2018). Ha sido consultor nacional e internacional en temas de gerencia pública y talento humano en el sector público. Líneas de investigación: formación en gestión pública, los procesos de modernización de la gestión pública en Chile y las Américas, y la ética y probidad de los funcionarios públicos. Publicaciones recientes: Sanabria, Pablo, Pliscoff, Cristian y Gomes, Ricardo, "E-Government Practices in South American Countries: Echoing a Global Trend or Really Improving Governance? The Experiences of Colombia, Chile, and Brazil", en Gascó-Hernández, Mila [ed.], Open Government: 14 (Public Administration and Information Technology), Estados Unidos: Springer (2014); Pliscoff, Cristian, "Servicio País: A Chilean Case of Professional Volunteering", en Bryer, Thomas [ed.], National Service and Volunteerism: Achieving Impact in Our Communities, Estados Unidos: Lexington Books (2014); Pliscoff, Cristian [comp.], Enseñanza y aprendizaje de administración y politicas públicas en las Américas, Chile: Santiago Ril editores (2014).

Recepción: 8 de julio de 2015.

Aprobación: 4 de octubre de 2016. 